\title{
Evaluation of Total Antioxidant Capacity in Serum and Follicular Fluid of Women Undergoing ICSI and its Association with Implantation Failure
}

\author{
Lana Nazar Abdul-Razzaq1, Khalid Mahdi Salih², Bushra Jawad Al-Musawi ${ }^{3}$ \\ ${ }^{1}$ Assist. Lecturer, Ministry of Education, ${ }^{2}$ Prof. Dr., Department of biology, \\ College of Science-Al-Mustansiriya University, ${ }^{3}$ Dr., Kamal AL-Samarrai Hospital, Baghdad, Iraq
}

\begin{abstract}
Purpose: Total antioxidant capacity (TAC) in women serum and follicular fluid (FF) which surrounding oocytes may be related to the implantation failure. Therefore, we herein examined the relationship between total antioxidant capacity status in serum and FFand it is association with implantation failure

Method: One hundred and seventeen of non-reproductive women who underwent intra-cytoplasmic sperm injection (ICSI)included in this study and conducted between March 2018 and April 2019 in Kamal ALSamarai Hospital, center of fertility and IVF. Serum and follicular fluid were collected from non-reproductive women aged ranged 20-45 years and BMI (ranged 21.9-36.3kg/ $\mathrm{m}^{2}$ ), TAC were measured using sandwich ELISA in serum and follicular fluid specimen of 21 women of successful implantation compared to 96 experienced implantation failures.

Results: TAC was increased in serum of implantation failure compared to successful but not significant differences between two study groups. Whereas TAC levels were highly significant $(\mathrm{P}=0.002)$ inFF of women who had successful implantation $(1.08 \pm 0.64 \mathrm{mmol} / \mathrm{L})$ whereas in failure were lower $(0.55 \pm$ $0.42 \mathrm{mmol} / \mathrm{L}$ ). In addition to that, when evaluating the frequency of TAC category in FF revealed highly significant differences $(\mathrm{P}=0.003)$ between two groups, the majority of failure groups $(84.4 \%)$ had low TAC compared with (40\%) in successful groups, while a significant increase of sufficient TAC in successful than failure groups (50\% versus $6.1 \%$ respectively), whereas the borderline TAC were $(9.1 \%$ versus $10 \%)$ in failure and successful groups.
\end{abstract}

Conclusions: TAC in FF may be potential markers for implantation successful in ICSI cycle.

Keywords: Follicular fluid,antioxidant,implantation failure, intra-cytoplasmic sperm injection.

\section{Introduction}

In fact, despite advances in assisted reproduction treatment, poor oocyte quality remains a subtle problem for female infertility, and the investigation of factors that affect IVF/ICSI outcome may help to improve success rates. FF is a serum transudate, which contains metabolism products by granulosa and theca cells and provides the micro-environment of the grown oocyte, directly influences on the oocyte quality and implantation ${ }^{1}$. Several studies have focused on the microenvironment surrounding the oocyte, such as ROS and antioxidants found in $\mathrm{FF}^{(1-2)}$. Oxidation stress has been suggested as one of the most important factors that negatively affect assisted reproduction outcome ${ }^{3}$, in order to protect the follicles from oxidative insult, follicular fluid is naturally provided with an efficient antioxidant system ${ }^{4}$. Total anti-oxidants status (TAS) is composed of antioxidant capacity of total protein (85\%; mainly albumin), uric acid, bilirubin, carotenoids, tocopherol and ascorbic acid 5 . Indeed, an imbalance between ROS and the antioxidant defense system in the FF could be responsible for abnormal oocyte development, causing damage to the DNA, cytoskeleton and cell membrane, which would result in lower egg quality and lead to decreased fertilization potential 
of the oocytes in ART cycles ${ }^{6}$. Most of losses of antioxidant in human reproduction take place even before the implantation as up to $50 \%$ of losses occur during that time. Also, the environment is influenced by endocrine signaling ${ }^{7}$ and by the type of gonadotrophin the follicle is exposed to during the follicular phase leading to reduced protectionagainst oxidation ${ }^{8}$. Therefore, the objective of this study was to determine the association between totalantioxidant capacity andimplantation rate, bothin serum and FF of women had successful implantation compared to those inwomen with implantation failure.

\section{Materials and Method}

\section{Subjects}

The study included 117women (mean age 31.1 \pm 5.7years)admitted atCenter of infertility diagnosis and assisted reproductive technology/Kamal ALSamarai Hospital. This cross-sectional study of nonreproductive women who underwent intra-cytoplasmic sperm injection consisting of 21 women with successful implantation and 96 women with implantation failure were recruited. Patients with endometriosis, endometrial polyps, fibroid in uterus and diabetes mellitusand any systemic disease were excluded. Indication for ICSI in non-reproductive women was tubal obstruction or male factor infertility.

Ovarian Stimulation Protocol: All of the patients received gonadotropin releasing hormone antagonist (GnRH-ant) protocol for ovarian stimulation and were treated with recombinant follicle-stimulating hormone (rFSH) (Gonal-F, Merckserono, Switzerland) per day from the 2nd day of spontaneous or induced menstruation. The dose of gonadotropins was adjusted according to ovarian response, as detected by ultrasound examination. As soon as the dominant follicle reached $14 \mathrm{~mm}$ in diameter, $(\mathrm{GnRH})$ antagonistcetrorelix at $0.25 \mathrm{mg}$ (Cetrotide $\AA$, Serono, Switzerland) was administered daily, until the day of ovulationtriggering which was obtained by hCG injection (Ovitrelle at $250 \mu \mathrm{g}$; Merck- Serono, Geneva: Switzerland), when atleast three follicles of size $>18 \mathrm{~mm}$ were present in the ovaries, oocyte puncture was performed at the 36th hour after hCG injection. After FF aspiration oocytes were separated and transferred into culture media, then, FF was located into a $15-\mathrm{ml}$ plane tube and centrifuged at $300 \mathrm{~g}$ for $5 \mathrm{~min}$ and supernatant was stored at $-80^{\circ} \mathrm{C}$ until further analysis; also for each patient, at the day of embryo transfer, blood serum samples for comparative analysis was collected.

ICSI Producers: Oocyte denudation and ICSI were performed 3 hours after retrieval, and the in vitro culture was carried out in cleavage Gain medium (Fertipro/Belgium) under mineral oil until day 2 (2-5 cells stage) in automated incubators with $6 \% \mathrm{CO} 2$ at $37^{\circ} \mathrm{C}$, the growth of all the embryos from each patient $(n=117)$ was continuously monitored. Embryo quality was assessed before embryo-transfer, and a maximum of three embryos transferred to all patients. Pregnancies were diagnosed by serum positive B-HCG levels $(>100$ $\mathrm{miu} / \mathrm{ml}) 14$ days after embryo transfer.

Parameters Analyses: Age, duration of nonreproductive, body mass index (BMI), serum E2 were assessed as possible confounders. E2 were measured sandwich enzyme immunoassay ELISA method based on a human monoclonal antibody (Biomerieux/France) according to the manufacturer's instructions. TAC was measured in serum and FF using a test kit (Omnignostix $\mathrm{GmbH} \& \mathrm{CoKH}$, Austria) by spectrophotometric quantification. Briefly, it is based on the reaction of peroxides with peroxidase followed by a color reaction of the chromogenic substrate tetra-methyl-benzidinein the presence of biological antioxidants. Its blue colour turns to yellow complex after addition of the stop solution which had a maximum absorbance at the wavelength of $450 \mathrm{~nm}$.

Statistical Analysis: Statistical analysis carried out by using Vassar Stats Web Site for Statistical Computation (Lowry, 2013). Qualitative data expressed as percentage values, whereas measurable data expressed as $(\mathrm{M} \pm$ $\mathrm{SE})$. However; the difference between two independent samples analyzed by t-test, while comparison of categorical data between the different groups carried-out by using Chi square test. The significance of differences estimated at two-tail P level less than 0.05 .

\section{Results}

Demographic and clinical characteristic parameters of the subjects are presented in Table 1. The ICSI cycle characteristics of our patients are shown in Table 2. The total number of retrieved oocytes was 1094 (range 1-28), at the time of the ICSI procedure, the nuclear maturity of the intact oocytes revealed 801 oocytes in metaphase II and ranged from 1-23, embryo obtained was 537. The mean percentage of efficiency of fertilization rate was $69 \%$, the mean ranged of embryo transfer was $1-5$, regarding implantation status, only twenty one of women 
has revealed successful implantation whereas nighty six women has implantation failure. The clinical ongoing implantation rate per transferred embryo was $17.9 \%$. Table 3 shows the antioxidant profile in serum and FF of two groups of women's. TAC were higher in serum of women who had implantation failure than successful but, did not show significant differences $(2.24 \pm 0.52$ versus $1.96 \pm 0.42 \mathrm{mmol} / \mathrm{L}$, respectively, $\mathrm{P}=0.051$ ). In contrast, TAC were decreased in FFof women who had implantation failure $(0.55 \pm 0.42 \mathrm{mmol} / \mathrm{L})$ compared to women of successful implantation $(1.08 \pm 0.64 \mathrm{mmol} / \mathrm{L})$ and show highly significant $(\mathrm{P}=0.002)$.

Table 1: Demographic and clinical characteristic of the patients.

\begin{tabular}{|l|lr|}
\hline Variables & Range & Mean \pm SD \\
\hline No. of patients & 117 & \\
\hline Age (years) & $20-45$ & $31.1 \pm 5.7$ \\
\hline Infertility duration (Years) & $2-24$ & $7.8 \pm 4.3$ \\
\hline Weight $(\mathrm{kg})$ & $48-108$ & $73.1 \pm 10.5$ \\
\hline Length $(\mathrm{m})$ & $1.43-1.78$ & $1.59 \pm 0.06$ \\
\hline BMI $\left(\mathrm{kg} / \mathrm{m}^{2}\right)$ & $21.9-36.3$ & $28.6 \pm 3.6$ \\
\hline E2 level $(\mathrm{pg} / \mathrm{ml})$ & $255-4023$ & $1583 \pm 895$ \\
\hline
\end{tabular}

BMI: body mass index; E2: estradiol.

Table 2: ICSI cycle characteristics of patients.

\begin{tabular}{|c|c|c|}
\hline Variables Total & Range & Average \\
\hline Retrieved oocyte 1094 & $1-28$ & 9.3 \\
\hline MII801 & $1-23$ & 6.8 \\
\hline Embryo obtained 537 & $0-18$ & 4.6 \\
\hline \multicolumn{3}{|c|}{ Efficiency of fertilization $69 \%$} \\
\hline Embryo transferred --- & $1-5$ & --- \\
\hline \multicolumn{3}{|l|}{ Implantation status } \\
\hline Successful 21 & --- & --- \\
\hline Failure 96 & -- & --- \\
\hline Implantation rate $17.9 \%$ & & \\
\hline
\end{tabular}

Table 3: Comparison of antioxidant status between successful and failure implantation groups

\begin{tabular}{|l|c|c|c|}
\hline \multirow{2}{*}{ Total anti-oxidant capacity $(\mathbf{m m o l} / \mathbf{L})(\mathbf{M} \pm$ SD) } & \multicolumn{2}{|c|}{ Implantation Group } & \multirow{2}{*}{ P value } \\
\cline { 2 - 4 } & Successful (n=21) & Failure (n=96) & 0.051 \\
\hline In serum & $1.96 \pm 0.42$ & $2.24 \pm 0.52$ & 0.002 \\
\hline In follicular fluid & $1.08 \pm 0.64$ & $0.55 \pm 0.42$ & \\
\hline TAC $<1$ (low); 1-1.3 (borderline); $>1.3$ (sufficient) & & & \\
\hline
\end{tabular}

Values are mean $\pm \mathrm{SD}$; TAC: Total antioxidant capacity.

In order to confirm these data, we evaluated the frequency of TAC category in FF as shown in Fig 1, and revealed highly significant differences $(\mathrm{P}=0.003)$. The majority of failure groups $(84.4 \%)$ had low TAC compared with $(40 \%)$ in successful groups, while a significant increase of sufficient TAC in successful than failure groups (50\% versus $6.1 \%$ respectively); in addition to that, the borderline TAC were $(9.1 \%$ versus $10 \%$ ) in failure and successful groups. 


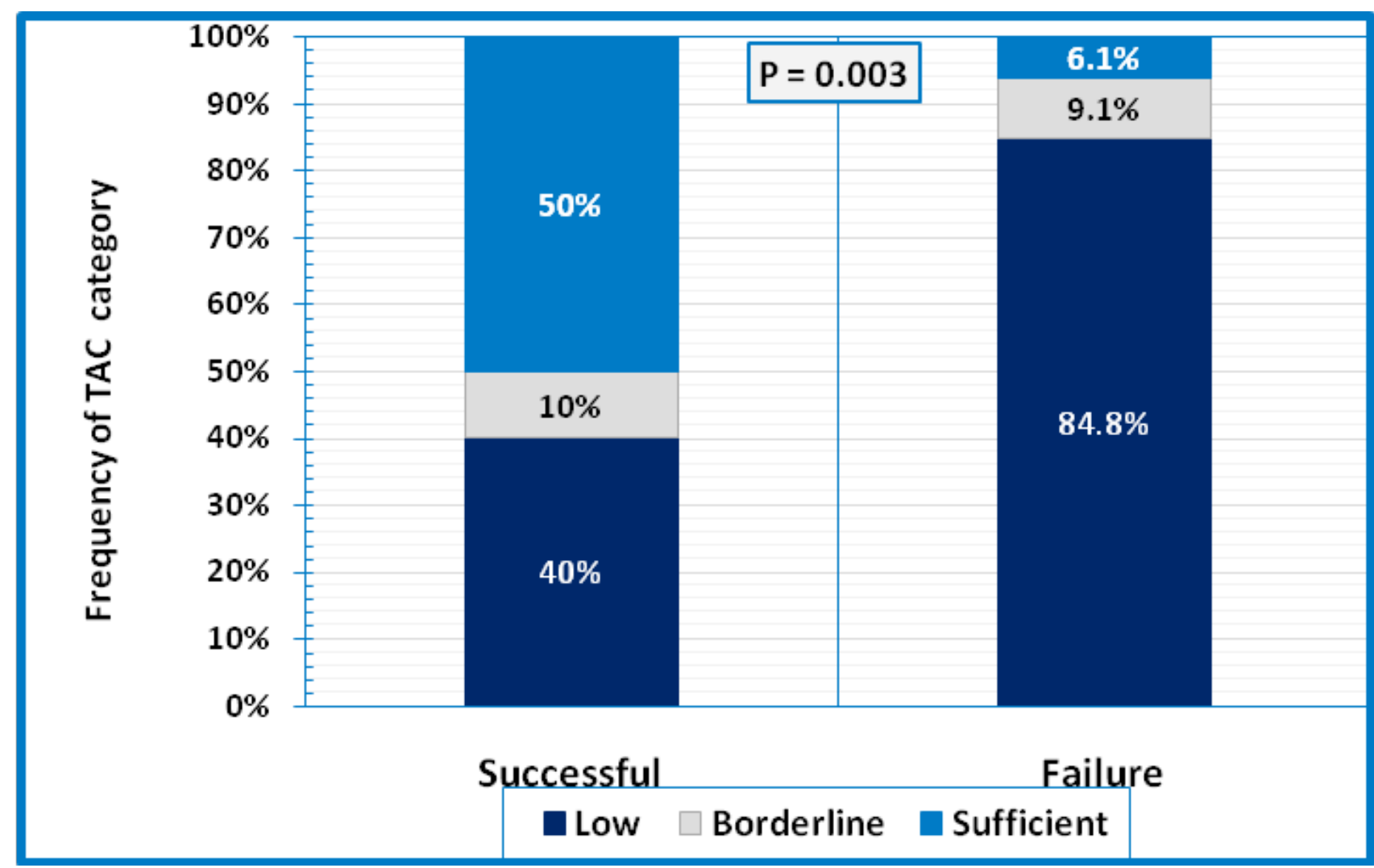

Figure 1. Frequency of TAC categories in follicular fluid of women with successful and failure implantation.

\section{Discussion}

In spite of the total number of oocyte retrieved and efficiency of fertilization was high, it appears that failure to achieve implantation with ICSI in this study was very high. Research about antioxidants status appears to be in strict relation with assisted reproduction outcome. In the present study, total TAC levels were lower in FF of patients who had implantation failure after ICSI. However, follicular fluid forms the biochemical micro-environment of the oocyte before ovulation and assists in estimating the developmental competence of female gametes ${ }^{1}$. FF contains proteins, sugars, ROS, antioxidants, and hormones which have a direct impact on the maturation ability and the quality of oocytes, also rich in low molecular weight metabolites that are direct or indirect regulators of oxidative stress and antioxidant production ${ }^{9}$. Rupture of the follicular wall during ovulation can be modeled as a short inflammatory process. An increase in various substances in the follicle near the time of ovulation, which can induce oxidative stress. Free radical-generating agents include histamine, bradykinim, angiotensin, prostaglandins (PG), eicosanoids, proteolytic enzymes, nitric oxide, and superoxide $^{10}$. The ROS are produced within the follicle during the ovulation process ${ }^{10}$, imbalance between antioxidants factors and ROS production in ovarian FF could adversely influence on the quality of the oocyte, fertilization, and embryo development ${ }^{11}$.Elevated ROS levels in patients with unexplained infertility imply reduced levels of antioxidants such as vitamin $\mathrm{E}$ and glutathione, resulting in a reduced ability to scavenge ROS and neutralize its toxic effects ${ }^{12}$. On the other hand, ROS could induce inflammatory response accompanied by the releasing of pro-inflammatory cytokines ${ }^{13}$ such as, IL-6 decreases aromatase activity within follicles, which lead to reduction in intra-follicular estradiol concentration, fertility and fertilizing capacity ${ }^{6}$. Inflammation and oxidative stress have been implicated in the pathogenesis of several chronic disorders ${ }^{14}$. Although ovarian stimulation also induces ROS production, disrupts the oxidant- antioxidant balance and leads to oxidative stress ${ }^{15}$. Our observation is an agreement with the literature reports have shown that women who became pregnant after IVF therapy had a tendency toward higher levels of TAC in their follicular fluid compared to those who did not achieve pregnancy 
16. Several research groups have concluded that the oxidant-antioxidant balance in the oocytes environment can have a significant impact on IVF outcome in women with endometriosis ${ }^{17}$. On the other hand, obtained in a study by Attaran et al. ${ }^{18}$ who investigated FF levels TAC in women undergoing IVF; but these authors did not observe a difference in TAC levels between patients who became pregnant and those who did not. In contrast, high TAC level has been reported as a marker for poor response to ovulation induction in women with polycystic ovarian syndrome ${ }^{19}$. On the basis of the etiology of infertility, women with male factor infertility, which can be considered as healthy control subjects, presented the best follicular antioxidant profile in comparison to those with female or unexplained infertility, confirming the presence of oxidation stress and reduced antioxidant capacity in FF from women with reproductive diseases 20. In accordance with this study, previous reports have shown that follicular total antioxidant capacity is positively correlated with pregnancy rate ${ }^{21}$; at the same time, a previous study demonstrated that elevated blood plasma antioxidant status was favorable for achieving clinical pregnancy ${ }^{20}$. In short, both systemic and local antioxidant status appears to be in strict relation with assisted reproduction outcome. The results may be help physicians on the treatment of IVF/ICSI, as well as scientists in clarifying the etiology of ICSI ${ }^{22}$.

Financial Disclosure: There is no financial disclosure.

Conflict of Interest: None to declare.

Ethical Clearance: All experimental protocols were approved under the Ministry of Education and all experiments were carried out in accordance with approved guidelines.

\section{References}

1. Revelli A, DellePiane L, Casano S, Molinari E, Massobrio M, Rinaudo P. Follicular fluid content and oocyte quality: from single biochemical markers to metabolomics. Reprod Biol Endocrinol. 2009; 7:40.

2. Fujimoto VY, Bloom MS, Huddleston HG, Shelley WB, Ocque AJ, Browne RW. Correlations of follicular fluid oxidative stress biomarkers and enzyme activities with embryo morphology parameters during in vitro fertilization. Fertil. Steril. 2011; 96:1357-1361.
3. Agarwal A, Aponte-Mellado A, Premkumar BJ Shaman A, Gupta S. The effects of oxidative stress on female reproduction: a review. Reprod. Biol. Endocrinol. 2012; 10:49.

4. Agarwal A, Gupta S, Sikka S. The role of free radicals and antioxidants in reproduction. Curr Opin Obstet Gynecol 2006; 18 (3):325-32.

5. Altindag O, Erel O, Soran N, Celik H, Selek S. Total oxidative/antioxidative status and relation to bone mineral density in osteoporosis. RheumatolInt 2008; 28:317-21.

6. Nunez-Calonge R, Cortes S, Gutierrez Gonzalez $\mathrm{LM}$, et al. Oxidative stress in follicular fluid of young women with low response compared with fertile oocyte donors. Reprod Biomed Online. 2016; 32:446-56.

7. Bristol SK, Woodruff TK. Follicle-restricted compartmentalization of transforming growth factor beta superfamily ligands in the feline ovary. Biol Reprod 2004; 70:846-859.

8. Aurrekoetxea I, Ruiz-Sanz JI, del Agua AR, Navarro R, Herna'ndez ML, Matorras R, Prieto B, Ruiz-Larrea MB. Serumoxidizability and antioxidant status in patientsundergoing in vitro fertilization. Fertil. Steril. 2010; 94:1279-1286.

9. Tamura H, Takasaki A, Miwa I, Taniguchi K, Maekawa R, Asada H, et al. Oxidative stress impairs oocyte quality and melatonin protects oocytes from free radical damage and improves fertilization rate. J Pineal Res. 2008; 44:280-7.

10. Sugino N. Reactive oxygen species in ovarian physiology. Reprod Med Biol 2005;4:31-44.

11. Yalcinkaya E, Cakiroglu Y, Doger E, et al. Effect of follicular fluid NO, MDA and GSH levels on in vitro fertilization outcomes. J Turkish German Gynecol Assoc 2013;14:136-41.

12. Wang Y, Sharma RK, Falcone T, Goldberg J, Agarwal A. Importance of reactive oxygen species in the peritoneal fluid of women with endometriosis or idiopathic infertility. FertilSteril 1997; 68(5):826-30.

13. Touyz RM. Molecular and cellular mechanisms in vascular injury inhypertension: role of angiotensin II - editorial review. Curr Opin Nephrol Hypertens 2005;14:125-31.

14. Biswas SK. Does the interdependence between oxidative stress andinflammation explain the antioxidant paradox?. Oxidat Med Cell Longev. 
2016;2016:5698931.

15. Palini S, Benedetti S, Tagliamonte MC, et al. Influence of ovarian stimulation for IVF/ICSI on the antioxidant defence system and relationship to outcome. Reprod Biomed Online 2014; 29:65-71.

16. Pasqualotto EB, Agarwal A, Sharma RK, Izzo VM, Pinotti JA, Joshi NJ and Rose BI. Effect of oxidative stress in follicular fluid on the outcome of assisted reproductive procedures. Fertil Steril.2004; 81(4):973-976.

17. Prieto L, Quesada JF, Cambero O, Pacheco A, Pellicer A, Codoceo R, et al. Analysis of follicular fluid and serum markers of oxidative stress in women with infertility related to endometriosis. FertilSteril. 2012; 98:126-30.

18. Attaran M, Pasqualotto E, Falcone T, Goldberg JM, Miller KF, Agarwal A and Sharma RK .The effect of follicular fluid reactive oxygen species on the outcome of in vitro fertilization. Int J FertilWomens Med. 2000;45:314-320.
19. FerdaVerit F, Erel O, Kocyigit A. Association of increased total antioxidant capacity and anovulation in nonobese infertile patients with clomiphene citrate-resistant polycystic ovary syndrome. FertilSteril. 2007;88:418-424.

20. Velthut A, Zilmer M, Zilmer K, Kaart T, Karro H, Salumets A. Elevated blood plasma antioxidant status is favourable for achieving IVF/ICSI pregnancy. Reprod Biomed Online. 2013; 26:34552

21. Bedaiwy MA, Elnashar SA, Goldberg JM, Sharma R, Mascha EJ, Arrigain S, Agarwal A, Falcone T, Effect of follicular fluid oxidative stress parameters on intracytoplasmic sperm injection outcome. Gynecol. Endocrinol. 2012;28:51-55.

22. Ozkaya MO, Naziroglu M, Barak C, Berkkanoglu M. Effects of multivitamin/mineral supplementation on trace element levels in serum and follicular fluid of women undergoing in vitro fertilization (IVF). Biol Trace Elem Res. 2011; 139:1-9. 